\title{
Insulin is a key determinant of elevated retinal arteriolar flicker response in insulin-resistant individuals
}

\author{
Manja Reimann ${ }^{1} \cdot$ Walthard Vilser ${ }^{2}$ - Matthias Gruber ${ }^{3}$ - Stefan R. Bornstein ${ }^{3}$. \\ Tjalf Ziemssen ${ }^{1}$
}

Received: 4 March 2015 / Accepted: 29 April 2015 /Published online: 24 May 2015

(C) Springer-Verlag Berlin Heidelberg 2015

\begin{abstract}
Aims/hypothesis Insulin may link metabolic disorders to retinal microvascular pathology. The aim of the present study was to investigate the impact of early insulin resistance on retinal microcirculation.

Methods Retinal diameter responses to flicker-light stimulation were investigated in 81 clinically healthy participants (32 \pm 6 years $[$ mean $\pm \mathrm{SD}], 59 \%$ women) who were recruited according to their BMI. All participants underwent an OGTT and euglycaemic-hyperinsulinaemic clamp $\left(40 \mathrm{mU} / \mathrm{m}^{2}\right.$. $\min ^{-1}$ insulin dose). After stratification by low and high insulin sensitivity based on a clamp-derived glucose disposal rate of $\leq$ or $>4.9 \mathrm{mg} / \mathrm{kg}$ body mass, respectively, baseline retinal diameters and their relative changes to flicker stimulation were compared while controlling for mean arterial pressure, BMI and sex.

Results The arterial vasodilator response at the end of flicker stimulation $(p=0.044)$ and the area under the arterial reaction curve during flicker stimulation $(p=0.015)$ were significantly higher in individuals with low vs high insulin sensitivity. Vasodilatory responses of retinal veins to flicker stimulation and baseline retinal diameters did not differ between insulinsensitive and insulin-resistant participants $(p>0.05)$. In a
\end{abstract}

Manja Reimann

manja.reimann@uniklinikum-dresden.de

1 Autonomic and Neuroendocrinological Laboratory Dresden, Department of Neurology, University Hospital Carl Gustav Carus, Technische Universität Dresden, Fetscherstr. 74, 01307 Dresden, Germany

2 Imedos Systems UG, Jena, Germany

3 Department of Internal Medicine, University Hospital Carl Gustav Carus, Technische Universität Dresden, Dresden, Germany stepwise linear regression analysis, fasting insulin remained the only predictor of the arterial vasodilator response to flicker-light $(p<0.01)$. Waist circumference also contributed, although to a lesser extent, to the arterial vasodilator response $(p=0.023)$.

Conclusions/interpretation Insulin sensitivity is an important determinant of retinal microvascular function. We propose that the elevated arterial flicker response in insulin-resistant states is a result of higher circulating insulin levels.

Keywords Insulin resistance $\cdot$ Nitric oxide $\cdot$ Retinal flicker response
Abbreviations
BM Body mass
GDR Glucose disposal rate
iNOS Inducible nitric oxide synthase
MAPK Mitogen-activated protein kinase
NO Nitric oxide
NOS Nitric oxide synthase
PI3K Phosphatidylinositol 3-kinase

\section{Introduction}

Microangiopathy is a common complication in diabetes mellitus and may contribute to the excess cardiovascular risk seen in patients with the disease. In numerous studies, retinal vascular signs have been repeatedly correlated with cardiovascular risk factors, incident cerebrovascular and cardiovascular outcomes, and diabetic microvascular complications [1-4]. These findings indicate that the retinal microcirculation may be an ideal site to study the proposed linkage between metabolic disorders and vascular complications. Structural and 
functional retinal abnormalities found in diabetes mellitus have been thought to reflect a range of subclinical pathophysiologic responses to obesity, hyperglycaemia, hypertension, inflammation and hypoxia [5-7]. Despite being considered a key event leading to type 2 diabetes mellitus, the contribution of insulin resistance to retinal microvascular changes has received little attention. Resistance to vascular actions of insulin has been found to contribute to the pathogenesis of macrovascular diabetic complications [8], while microvascular complications have frequently been considered secondary to hyperglycaemia. Evidence is now accumulating that insulin resistance may also play a pathophysiological role in the development of microvascular complications. Previously, it has been shown that microvascular dysfunction in lean and obese women is related to impaired insulin sensitivity [9]. A recent study in patients with type 1 diabetes mellitus found an independent relationship between insulin resistance and diabetic microangiopathy [10]. Another study revealed that individuals at risk for diabetes have similar attenuated retinal microvascular responses to flicker-light to those with type 2 diabetes mellitus, indicating that retinal microvascular changes are present before the development of manifest diabetes and associated complications [7].

To shed light on the possible aetiological role of insulin resistance in the development of microvascular abnormalities, we studied retinal vasodilator responses to flicker-light stimulation in clinically healthy individuals who were stratified into insulin-sensitive and insulin-resistant groups.

\section{Methods}

Study design and population The current Influence of Adiposity and Insulin resistance on Cardiovascular and Autonomic Integrity (LAICA) study investigated 98 white participants (40\% men, $60 \%$ women) aged $32 \pm 6$ (mean $\pm \mathrm{SD}$ ) years between 2009 and 2014. Participants were recruited from the metropolitan area of Dresden by online and newspaper advertisements. To be eligible, participants were clinical healthy and between 25 and 45 years of age with a BMI $\geq 20 \mathrm{~kg} / \mathrm{m}^{2}$. The health state of each participant was verified during a screening visit by assessing a complete medical history and by physical and laboratory examinations (full biochemical profile). Supine resting BP and 12-lead electrocardiogram was recorded by the Finometer device (FMS Finapress Medical Systems, Amsterdam, the Netherlands) and the ELI 150 Px electrograph (Mortara Instrument, Milwaukee, WI, USA). No participants were taking any medications or dietary supplements. Exclusion criteria included arrhythmia, atrioventricular block, hypertension, peripheral or autonomic neuropathy, cardiovascular, cerebrovascular, thyroid, renal, or liver disease, epilepsy, increased ocular pressure $(>21 \mathrm{mmHg})$, ocular disease, pregnancy and current or anticipated weight- reduction diet. Upon eligibility, participants were stratified into one of three BMI groups based on the criteria of WHO for normal weight, overweight and obese individuals [11]. The purpose of this stratification was to obtain a large spectrum of insulin sensitivity. Study procedures were performed in line with the Declaration of Helsinki, and the study protocol was approved by the Ethics Committee of the Faculty of Medicine of the Technische Universität Dresden. All participants provided informed written consent.

Anthropometry and body composition Height was reported by the participants. Metabolic weight was measured with a digital scale. BMI was calculated as body mass (BM) in kilograms divided by height in metres squared. Whole-body per cent body fat was measured by TANITA Body Composition Analyzer Type TBF-300MA (Tanita Europe BV, Amsterdam, the Netherlands), and fat mass and lean body mass were calculated by integrated algorithms. Body surface area was calculated using the $\mathrm{Du}$ Bois equation: $0.007184 \times$ height $(\mathrm{cm})^{0.725} \times \mathrm{BM}(\mathrm{kg})^{0.425}$. Waist circumferences were measured using a conventional measuring tape.

\section{Assessment of glucose tolerance and insulin sensitivity}

Participants attended the metabolic ward on two separate mornings, having fasted for $12 \mathrm{~h}$ and abstained from caffeine and alcohol for $24 \mathrm{~h}$. They were instructed not to exercise on the day prior to investigations to eliminate acute effects of exercise on plasma glucose levels. Diagnostic tests were performed in a quiet room (ambient temperature $22^{\circ} \mathrm{C}$ ) with the participants sitting (OGTT) or lying in a supine position (euglycaemic clamp) after having rested for $30 \mathrm{~min}$. Glucose tolerance was evaluated during an OGTT by administering a $75 \mathrm{mg}$ glucose bolus diluted in tea. Blood samples for analysis of plasma glucose and insulin levels were taken via an indwelling catheter at timed intervals $(0,30,60,90$ and $120 \mathrm{~min})$. HOMA-IR was calculated by the following equation: [fasting insulin $(\mathrm{pmol} / \mathrm{l}) \times$ fasting glucose $(\mathrm{mmol} / \mathrm{l})] / 135$. In vivo insulin sensitivity was assessed using the de Fronzo (1979) euglycaemic-hyperinsulinaemic clamp technique with a primed continuous infusion of insulin at $40 \mathrm{mU} \mathrm{m}^{-2} \mathrm{~min}^{-1}$ to achieve endogenous steady-state insulin concentrations and to draw steady-state blood samples [12]. An intravenous catheter was placed in the antecubital vein for infusion of insulin and glucose. A second catheter was placed retrograde in a dorsal vein of the contralateral hand for blood sampling. The hand was wrapped with a heating pad for arterialisation of venous blood. Blood glucose was clamped at $5.5 \mathrm{mmol} / \mathrm{l}$ by infusion of a $20 \%$ glucose solution at varying rates according to current blood glucose concentrations, measured every 5 min (Biosen C_line sport glucose auto-analyser; EKF-Diagnostic, Barleben, Germany). The mean glucose infusion rate during steady-state, defined as relative stability of blood glucose concentration (usually between 60 and $120 \mathrm{~min}$ ), was used to 
calculate whole-body glucose uptake (steady-state glucose utilisation adjusted for BM). Steady-state plasma insulin was measured at $10 \mathrm{~min}$ intervals and steady-state values were averaged to calculate steady-state glucose disposal rate (GDR) adjusted for BM and steady-state insulin concentration.

Biochemical profile All biochemical analyses were performed at the Institute of Clinical Chemistry and Laboratory Medicine of the University Hospital Carl Gustav Carus, Dresden. To validate that participants were free of subclinical disease, a comprehensive biochemical profile was obtained at the screening visit. Biochemical profiling included a small haemogram, hepatic enzymes, uric acid and bilirubin to evaluate liver function, urea and creatinine to evaluate renal function, thyroid-stimulating hormone and thyroid hormones to evaluate thyroid function, serum electrolytes, total plasma protein, C-reactive protein, IL-6, coagulation factors, glucose and a complete lipid profile. Blood samples obtained during the OGTT and the insulin clamp were analysed for glucose and insulin. All biochemical analyses were performed using validated and standardised analytical methods.

Retinal vascular imaging Following mydriasis of the right pupil by $1 \%$ tropicamide eye drops, the mean diameter of a retinal arterial and venous segment was continuously measured in relation to time and position using the Dynamic Vessel Analyzer (DVA; Imedos, Jena, Germany) [13]. Vessel segments of approximately $1.5 \mathrm{~mm}$ in length located between one and two disc diameters from the margin of the optic disc edge were examined in continuous light for $30 \mathrm{~s}$ followed by three cycles of $20 \mathrm{~s}$ flicker-light provocation only interrupted by $50 \mathrm{~s}$ of steady fundus illumination. Flicker-light was generated by an optoelectronic shutter that interrupted the green measuring light (530-600 nm, illumination of fundus approximately $6500 \mathrm{lux}$ ) with a frequency of $12.5 \mathrm{~Hz}$ over the entire $30^{\circ}$ image field, providing a sequence of one normal illuminated and one single dark frame at a video frequency of $25 \mathrm{~Hz}$. The integrated VesselMap 2 software version 3.1 (Imedos) allowed for automatic calculation of baseline diameters and maximal dilation. An interval of $30 \mathrm{~s}$ before the flicker stimulation was considered as baseline to which the subsequent diameter response was normalised. The maximal dilation was the largest vessel diameter at the end of each flicker stimulation ( $\pm 2 \mathrm{~s})$ averaged across three flicker periods. Due to large inter-individual variation, the maximal constriction was manually derived from averaged temporal responses as the absolute minimum of the arterial diameter after the end of flicker stimulation. Both maximal dilation and maximal constriction were expressed as per cent change over baseline values. The peak-to-peak value was manually calculated as the difference between the maximal and minimal arterial diameters. The area under the reaction curve during flicker stimulation was calculated in $[\% \times \mathrm{s}]$ as described previously by Kotliar et al [14]. The purpose was to assess differences in the curve form during flicker stimulation.

Statistical analysis Descriptive data are presented as mean $(95 \% \mathrm{CI})$ unless stated otherwise. Statistical analysis was performed with SPSS software package version 22.0 for Windows (SPSS, Chicago, IL, USA). All analyses were stratified according to insulin sensitivity as defined by clamp-derived GDR normalised for BM. We used the cut-off as proposed by Tam et al where a GDR $\leq 4.9 \mathrm{mg}[\mathrm{kg} \mathrm{BM}]^{-1} \mathrm{~min}^{-1}$ is indicative of insulin resistance (low insulin sensitivity) [15]. The $\chi^{2}$ test was used to test significance of group differences for sex. Oneway ANOVA was applied to test for differences in major group characteristics. Analyses of covariance were used to examine differences in retinal flicker variables between insulin-resistant and insulin-sensitive participants independent of mean arterial pressure, BMI and sex. Linear regression analyses were performed to determine relationships of discriminative retinal variables and selected markers of the insulin resistance syndrome. Statistical significance was accepted at $p<0.05$.

\section{Results}

Ten participants experienced health problems during the clamp and retinal vessel analysis of seven participants was of poor quality, consequently leading to exclusion from data analysis. A total of 81 participants remained of whom 19 were identified as having insulin resistance (Table 1). The insulinresistant group differed from insulin-sensitive participants in terms of haemodynamic, metabolic and anthropometric measures. The insulin-resistant group had a higher BMI, waist circumference and percentage body fat as well as higher fasting lipid, glucose and insulin concentrations. Mean arterial pressure was also significantly higher in the insulin-resistant group, with no differences in pulse pressure. According to the OGTT, six participants had impaired glucose tolerance. None was diagnosed with impaired fasting glucose and/or type 2 diabetes mellitus.

Mean maximal arterial dilation at the end of flicker stimulation and the area under the arterial reaction curve during the flicker were significantly higher in the insulin-resistant than the insulin-sensitive group, with no differences in baseline diameter of the analysed vessel segment (Table 2). There was a trend for reduced arterial constriction after cessation of the flicker in participants with insulin resistance as compared with insulin-sensitive participants. Retinal venous responses to flicker stimulation did not differ between the groups. Differences in retinal arterial responses to flickerlight were independent of differences in mean arterial pressure, BMI and sex distribution. 
Table 1 Baseline characteristics of study participants according to insulin sensitivity

\begin{tabular}{lllr}
\hline \multirow{2}{*}{ Characteristic $(n=81)$} & \multicolumn{2}{l}{ Insulin sensitivity $\left(\mathrm{GDR} \operatorname{mg}[\mathrm{kg} \mathrm{BM}]^{-1} \mathrm{~min}^{-1}\right)$} & \multirow{2}{*}{$p$ value } \\
\cline { 2 - 4 } & High $(>4.9)$ & Low $(\leq 4.9)$ & \\
\hline Sex male/female, $n$ & $26 / 36$ & $7 / 12$ & 0.693 \\
BMI $\left(\mathrm{kg} / \mathrm{m}^{2}\right)$ & $27(25,28)$ & $33(31,35)$ & $<0.001$ \\
Waist circumference $(\mathrm{cm})$ & $93(90,96)$ & $108(99,116)$ & $<0.001$ \\
Body fat (\%) & $29(27,32)$ & $38(35,42)$ & $<0.001$ \\
Triacylglycerol (mmol/l) & $1.1(0.9,1.2)$ & $1.6(1.1,2.0)$ & 0.003 \\
Total/HDL-cholesterol (mmol/l) & $3.1(2.9,3.4)$ & $3.9(3.5,4.3)$ & 0.003 \\
Fasting glucose (mmol/l) & $4.8(4.7,4.9)$ & $4.8(4.6,4.9)$ & 0.636 \\
120 min glucose (mmol/l) & $5.26(4.94,5.59)$ & $6.41(5.78,7.03)$ & 0.001 \\
Impaired glucose tolerance, $n(\%)$ & $3(4.8 \%)$ & $3(15.8 \%)$ & 0.111 \\
Fasting insulin (pmol/l) & $50.8(42.9,58.7)$ & $94.2(77.2,111.2)$ & $<0.001$ \\
120 min insulin (pmol/l) & $266(217,314)$ & $464(311,617)$ & 0.001 \\
HOMA-IR & $1.5(1.3,1.8)$ & $2.8(2.2,3.3)$ & $<0.001$ \\
Mean arterial pressure (mmHg) & $93(91,95)$ & $98(93,103)$ & 0.040 \\
Pulse pressure (mmHg) & $49(47,52)$ & $51(48,55)$ & 0.390 \\
\hline
\end{tabular}

Data are mean $(95 \% \mathrm{CI})$ unless otherwise indicated
Linear regression analysis indicated that insulin resistance syndrome markers (fasting insulin, mean arterial pressure, postprandial 120 min glucose, waist circumference, triacylglycerol, total cholesterol/HDL-cholesterol ratio) explained $21 \%$ of the variation in mean maximal arterial dilation in response to flicker (Table 3). From all entered variables, fasting insulin remained the only significant predictor of the arterial flicker response in a stepwise procedure. All entered insulin resistance syndrome markers explained $16 \%$ of variation in the area under the arterial flicker curve; however, this model did not reach statistical significance. In a stepwise regression analysis, fasting insulin and waist circumference were the only predictors of the area under the arterial flicker curve, each contributing $7 \%$ to its variation.

\section{Discussion}

The major observation of the present study is that insulinresistant individuals show elevated vasodilator responses of retinal arteries to flicker-light stimulation compared with insulin-sensitive individuals. These differences were independent of major confounders such as BMI, mean arterial pressure and sex. Baseline insulin status was independently

Table 2 Variables of retinal responses to flicker stimulation by insulin sensitivity

\begin{tabular}{|c|c|c|c|}
\hline \multirow[t]{2}{*}{ Variable $(n=81)$} & \multicolumn{2}{|c|}{ Insulin sensitivity (GDR $\mathrm{mg}[\mathrm{kg} \mathrm{BM}]^{-1} \min ^{-1}$ ) } & \multirow[t]{2}{*}{$p$ value } \\
\hline & $\operatorname{High}(>4.9)$ & Low $(\leq 4.9)$ & \\
\hline \multicolumn{4}{|l|}{ Retinal artery } \\
\hline Absolute diameter of the measured segment at baseline (MU) & $121(116,125)$ & $120(111,128)$ & 0.860 \\
\hline Mean maximal dilation in response to flicker ( $\%$ to baseline) & $4.6(3.9,5.3)$ & $6.3(4.9,7.6)$ & 0.044 \\
\hline Mean maximal constriction after flicker stimulation ( $\%$ to baseline) & $-1.9(-2.4,-1.4)$ & $-0.9(-1.9,0.01)$ & 0.084 \\
\hline Time of maximal vessel constriction (s) & $65(64,67)$ & $65(62,67)$ & 0.668 \\
\hline Peak-to-peak value $(\%)$ & $6.5(5.6,7.4)$ & $7.2(5.5,8.9)$ & 0.556 \\
\hline Area under the reaction curve during flicker stimulation $(\% \times \mathrm{s})$ & $52(42,63)$ & $83(62,105)$ & 0.015 \\
\hline \multicolumn{4}{|l|}{ Retinal vein } \\
\hline Absolute diameter of the measured segment at baseline (MU) & $152(147,158)$ & $148(138,159)$ & 0.497 \\
\hline Mean maximal dilation in response to flicker ( $\%$ to baseline) & $4.5(3.9,5.0)$ & $5.1(4.0,6.1)$ & 0.382 \\
\hline Area under the reaction curve during flicker stimulation $(\% \times \mathrm{s})$ & $67(58,76)$ & $71(53,89)$ & 0.697 \\
\hline
\end{tabular}

Data are mean $(95 \% \mathrm{CI})$ adjusted for sex, BMI and mean arterial pressure $\mathrm{MU}$, measuring units 
Table 3 Association of arterial flicker response with selected markers of the insulin resistance syndrome

\begin{tabular}{|c|c|c|c|c|c|c|c|}
\hline \multirow[t]{2}{*}{ Variable $(n=81)$} & \multirow[t]{2}{*}{$\mathrm{SD}$} & \multicolumn{3}{|c|}{$\begin{array}{l}\text { Maximal arterial flicker dilation }{ }^{\mathrm{a}} \\
\Delta R^{2}=0.21(p=0.034)\end{array}$} & \multicolumn{3}{|c|}{$\begin{array}{l}\text { Area under the arterial flicker curve } \\
\Delta R^{2}=0.16(p=0.109)\end{array}$} \\
\hline & & St. $\beta(95 \% \mathrm{CI})$ & $p$ value & Partial $r$ & St. $\beta(95 \% \mathrm{CI})$ & $p$ value & Partial $r$ \\
\hline \multicolumn{8}{|l|}{ All variables } \\
\hline Fasting insulin (pmol/l) & 36.9 & $0.57(0.26,0.92)$ & 0.001 & 0.429 & $0.52(0.15,0.84)$ & 0.003 & 0.377 \\
\hline Mean arterial pressure $(\mathrm{mmHg})$ & 9.07 & $-0.15(-1.31,0.19)$ & 0.235 & -0.153 & $-0.07(-0.31,0.19)$ & 0.617 & -0.066 \\
\hline 120 min glucose $(\mathrm{mmol} / \mathrm{l})$ & 1.36 & $0.16(1.37,0.18)$ & 0.210 & 0.161 & $0.11(-0.15,0.38)$ & 0.432 & 0.104 \\
\hline Waist circumference (cm) & 14.3 & $-0.17(-1.21,0.23)$ & 0.248 & -0.149 & $-0.36(-0.65,-0.02)$ & 0.024 & -0.295 \\
\hline Triacylglycerol (mmol/l) & 0.68 & $-0.01(-0.17,0.87)$ & 0.962 & -0.006 & $-0.06(-0.37,0.22)$ & 0.694 & -0.052 \\
\hline Total/HDL-cholesterol & 0.94 & $-0.10(-0.52,0.61)$ & 0.548 & -0.078 & $-0.02(-0.28,0.35)$ & 0.900 & -0.017 \\
\hline \multicolumn{8}{|l|}{ Stepwise regression $^{\mathrm{c}}$} \\
\hline Fasting insulin (pmol/l) & 36.9 & $0.40(0.15,0.63)$ & 0.001 & 0.396 & $0.47(0.15,0.79)$ & 0.002 & 0.378 \\
\hline Waist circumference (cm) & 14.3 & - & - & - & $-0.34(-0.62,-0.20)$ & 0.023 & -0.286 \\
\hline
\end{tabular}

Results are shown as standardised beta values (St. $\beta)(95 \% \mathrm{CI})$ and partial correlation coefficients $(r)$

${ }^{\mathrm{a}} \mathrm{SD}=2.70(\%)$

${ }^{\mathrm{b}} \mathrm{SD}=42.2(\% \times \mathrm{s})$

${ }^{\mathrm{c}}$ Maximal arterial flicker dilation $\Delta R^{2}=0.16$; area under the arterial flicker curve $\Delta R^{2}=0.15$

associated with the arterial flicker response and was revealed as a key determinant of arterial flicker dilation. Our findings provide evidence that insulin is implicated in retinal vascular changes in insulin-resistant states.

The retinal microcirculation is well suited for studying the association between insulin resistance and vascular function as it has been shown to be compromised over a wide hyperglycaemic continuum, thereby possibly contributing to the development of diabetic retinopathy and vision loss $[5,7$, 14]. Against previous evidence in healthy obese [14] and individuals with moderately elevated $\mathrm{HbA}_{1 \mathrm{c}}$ [7], our results indicate that the hyperaemic flicker response of retinal arteries is not reduced but heightened in early insulin resistance. It appears that previous findings were either not adjusted for differences in mean arterial pressure or were confounded by concomitant intake of statins and antihypertensive drugs, which are known to influence retinal diameters and flicker responses $[16,17]$. Tentative support for our findings can be found in a recent pilot study, in which flicker-stimulated retinal blood flow tended to be greater in insulin-resistant than insulinsensitive individuals [18].

Retinal vasodilation to flicker-light is a physiological response to meet enhanced metabolic requirements of stimulated neural cells (neurovascular coupling). Potassium and nitric oxide (NO) appear to be putative mediators of the hyperaemic response to flicker-light in healthy humans $[19,20]$ although this concept has previously been challenged by animal data [21]. Recent evidence in humans, however, renders a proposed role of epoxyeicosatrienoic acids and prostaglandins in vasodilation induced by flicker-light unlikely [22]. As glial cells and neurons are also capable of generating NO by the neuronal isoform of NO synthase (NOS) [23], the response of retinal vessels to flicker-light may be determined by both intact vascular endothelium and intact neuron-to-glial signalling. Recent findings suggest that the reduced retinal flicker response in early diabetes mellitus is a consequence of altered endothelial function rather than impaired neural activity [20]. Applied to our results, we conclude that early insulin resistance is associated with undisturbed retinal endothelial function. Although this conclusion contradicts the generally accepted view that retinal endothelial dysfunction links retinal vascular changes to diabetic complications [4], there is evidence that NO production in diabetes mellitus is not impaired but may even be increased [24-26]. Elevated NO production in diabetes mellitus has been related to upregulation of the inducible isoform of NOS (iNOS) in not only skeletal muscle but also retinas of individuals with diabetes mellitus [26, 27]. Experimental studies indicate that iNOS may contribute to the development of diabetic retinopathy and other vascular alterations in diabetes mellitus, independent of endothelial and neuronal isoforms [27]. Although we did not measure the central retinal arterial equivalent (an integrated measure of the diameter of all retinal arteries), our data do not support the probability that the elevated flicker response in insulin resistance reflects underlying changes in baseline arterial diameter.

Diminished sensitivity to insulin's vascular actions is thought to contribute to the pathophysiology of insulinresistant states [28]. Under physiological conditions, insulin augments blood flow in insulin-sensitive tissues primarily via phosphatidylinositol 3-kinase (PI3K) signalling that regulates endothelial NOS activity. In the ophthalmic artery, insulin 
may account for a $10 \%$ increase in choroidal blood flow; an effect that is completely abolished by NOS inhibition [29]. However, there is evidence that insulin may also stimulate alternative pathways of NO synthesis that involve the insulin-like growth factor-1 receptor [30]. This pathway may become particularly important in insulin resistance when insulin receptors are desensitised [31]. In insulin resistance, insulin has been found to promote vasoconstriction and proliferation due to a relative shift of vasodilatory $\mathrm{PI} 3 \mathrm{~K}-\mathrm{NO}$ signalling to vasoconstrictive mitogen-activated protein kinase (MAPK)-endothelin-1 signalling [32]. Although these molecular changes seem to contradict our findings, mechanistic studies have shown that MAPK pathways are crucially involved in the upregulation of iNOS gene expression in skeletal muscle and glial cells [33]. We propose that elevated circulating levels of insulin in insulin resistance may shift NO production from constitutive to inducible NOS isoforms that persistently produce higher amounts of $\mathrm{NO}$ once activated.

The pattern of changes in retinal vascular diameters in insulin-resistant states may, however, vary with the degree of hyperinsulinaemia and the presence of concomitant hyperglycaemia and other comorbidities [1,34]. As our study group was free of fasting hyperglycaemia, our results may primarily reflect retinal vascular changes in early stages of insulin resistance before manifestation of dysglycaemic conditions. The absence of hyperglycaemia may also explain why retinal venous responses to flicker-light were not different in insulin-resistant vs insulin-sensitive groups, although retinal venous widening and reduced venous dilation to flicker-light have previously been related to diabetes mellitus and diabetic microvascular complications $[35,36]$. It has been suggested that increases in blood flow and inflammation associated with hyperglycaemia and retinal hypoxia may be primarily responsible for changes in retinal venous function and thus may subsequently develop with the onset of chronic dysglycaemic conditions [37]. However, a previous study also demonstrated reduced retinal venous responses to flicker-light in obesity, although the study did not control for significantly higher glucose levels in the obese individuals [14]. In our study, insulin-sensitive and insulin-resistant individuals had comparable fasting glucose levels, which may additionally explain undisturbed retinal venous flicker responses.

A major limitation of our study is that we only analysed a single retinal artery and vein, which is not fully equivalent to the integrated calibre of all individual retinal arteries and veins. Another disadvantage is the lack of uniform reference values for the definition of insulin resistance from euglycaemic-hyperinsulinaemic clamps. Different thresholds of GDR have been established that vary as a function of BM, fat-free mass, body surface area or metabolic size $[15,38]$. We favoured GDR normalised for BM because it showed the strongest associations with retinal measures in the total sample (data not shown). Although clinical estimates of insulin resistance from routinely measured anthropometric and biochemical markers have similar sensitivity and specificity as GDR [38], they constitute only indirect measures of insulin sensitivity and were usually developed on the basis of clamp studies $[15,38]$. Impaired glucose tolerance was detected in six participants, whereas only three of them were found to have low insulin sensitivity according to the GDR cut-off. This difference may reflect limitations of the cut-off's sensitivity (67\%) and specificity (64\%) [15]. When excluding participants with impaired glucose tolerance from the insulinsensitive group the results did not change, although significance slightly weakened. Therefore, a partial contribution of prolonged postprandial hyperglycaemia to changes in retinal haemodynamics may also be considered.

In summary, we have demonstrated that retinal arterial dilation to flicker-light is enhanced in insulin-resistant as compared with insulin-sensitive individuals. Fasting insulin was identified as the only independent predictor of this response. We propose that the observed relationship between insulin and retinal vasodilator function reflects altered NOS regulation in insulin resistance.

Acknowledgements We are grateful to all volunteers for their participation. We thank B. Kindel and K. Haink (University Hospital Carl Gustav Carus, Dresden, Germany) for their kind technical assistance. We express our gratitude to J. Viehweg, K. Löbmann and A. Kästner (Technische Universität Dresden, Dresden, Germany) for their support in the study organisation and recruitment.

Funding This study was supported by the University Hospital Carl Gustav Carus, Dresden and the German Diabetes Foundation (271-07-10).

Duality of interest WV is chief executive officer of Imedos Systems UG (Jena, Germany) and a freelance researcher. All other authors declare that there is no duality of interest associated with their contribution to this manuscript.

Contribution statement MR is responsible for the study design, supervision, data collection, analysis and interpretation of data and drafting of the article. TZ contributed to the study design. MG and SRB contributed to medical supervision and data acquisition. WV contributed to data analysis and interpretation. All authors revised the manuscript for important intellectual content and gave final approval of the current version to be published. MR is the guarantor of this work and, as such, had full access to all the data in the study and takes responsibility for the integrity of the data and the accuracy of the data analysis.

\section{References}

1. Nguyen TT, Wong TY (2006) Retinal vascular manifestations of metabolic disorders. Trends Endocrinol Metab 17:262-268

2. Ding J, Wai KL, McGeechan K et al (2014) Retinal vascular caliber and the development of hypertension: a meta-analysis of individual participant data. J Hypertens 32:207-215 
3. Cheung CY, Tay WT, Ikram MK et al (2013) Retinal microvascular changes and risk of stroke: the Singapore Malay Eye Study. Stroke 44:2402-2408

4. Ikram MK, Cheung CY, Lorenzi M et al (2013) Retinal vascular caliber as a biomarker for diabetes microvascular complications. Diabetes Care 36:750-759

5. Mandecka A, Dawczynski J, Blum M et al (2007) Influence of flickering light on the retinal vessels in diabetic patients. Diabetes Care 30:3048-3052

6. Lim M, Sasongko MB, Ikram MK et al (2013) Systemic associations of dynamic retinal vessel analysis: a review of current literature. Microcirculation 20:257-268

7. Lott ME, Slocomb JE, Shivkumar V et al (2013) Impaired retinal vasodilator responses in prediabetes and type 2 diabetes. Acta Ophthalmol 91:e462-e469

8. DeFronzo RA, Ferrannini E (1991) Insulin resistance. A multifaceted syndrome responsible for NIDDM, obesity, hypertension, dyslipidemia, and atherosclerotic cardiovascular disease. Diabetes Care 14:173-194

9. de Jongh RT, Serne EH, IJzerman RG, de Vries G, Stehouwer CD (2004) Impaired microvascular function in obesity: implications for obesity-associated microangiopathy, hypertension, and insulin resistance. Circulation 109:2529-2535

10. Uruska A, Araszkiewicz A, Zozulinska-Ziolkiewicz D, Uruski P, Wierusz-Wysocka B (2010) Insulin resistance is associated with microangiopathy in type 1 diabetic patients treated with intensive insulin therapy from the onset of disease. Exp Clin Endocrinol Diabetes $118: 478-484$

11. World Health Organisation (2000) Obesity: preventing and managing the global epidemic. Report of a WHO consultation. World Health Organ Tech Rep Ser 894:1-253

12. DeFronzo RA, Tobin JD, Andres R (1979) Glucose clamp technique: a method for quantifying insulin secretion and resistance. Am J Physiol 237:E214-E223

13. Vilser W, Nagel E, Lanzl I (2002) Retinal vessel analysis- new possibilities. Biomed Tech (Berl) 47(Suppl 1 Pt 2):682-685

14. Kotliar KE, Lanzl IM, Schmidt-Trucksass A et al (2011) Dynamic retinal vessel response to flicker in obesity: a methodological approach. Microvasc Res 81:123-128

15. Tam CS, Xie W, Johnson WD, Cefalu WT, Redman LM, Ravussin E (2012) Defining insulin resistance from hyperinsulinemiceuglycemic clamps. Diabetes Care 35:1605-1610

16. Nagel E, Vilser W, Lanzl I (2004) Age, blood pressure, and vessel diameter as factors influencing the arterial retinal flicker response. Invest Ophthalmol Vis Sci 45:1486-1492

17. Reimann M, Prieur S, Lippold B et al (2009) Retinal vessel analysis in hypercholesterolemic patients before and after LDL apheresis. Atheroscler Suppl 10:39-43

18. Forst T, Weber MM, Mitry M et al (2012) Pilot study for the evaluation of morphological and functional changes in retinal blood flow in patients with insulin resistance and/or type 2 diabetes mellitus. J Diabetes Sci Technol 6:163-168

19. Dorner GT, Garhofer G, Kiss B et al (2003) Nitric oxide regulates retinal vascular tone in humans. Am J Physiol Heart Circ Physiol 285:H631-H636

20. Lasta M, Pemp B, Schmidl D et al (2013) Neurovascular dysfunction precedes neural dysfunction in the retina of patients with type 1 diabetes. Invest Ophthalmol Vis Sci 54:842-847
21. Metea MR, Newman EA (2006) Glial cells dilate and constrict blood vessels: a mechanism of neurovascular coupling. J Neurosci 26:2862-2870

22. Noonan JE, Dusting GJ, Nguyen TT et al (2014) Flicker lightinduced retinal vasodilation is unaffected by inhibition of epoxyeicosatrienoic acids and prostaglandins in humans. Invest Ophthalmol Vis Sci 55:7007-7013

23. Attwell D, Buchan AM, Charpak S, Lauritzen M, Macvicar BA, Newman EA (2010) Glial and neuronal control of brain blood flow. Nature 468:232-243

24. Dorner GT, Garhofer G, Selenko N et al (2003) The ocular hemodynamic response to nitric oxide synthase inhibition is unaltered in patients with early type I diabetes. Graefes Arch Clin Exp Ophthalmol 241:619-624

25. Schmetterer L, Findl O, Fasching P et al (1997) Nitric oxide and ocular blood flow in patients with IDDM. Diabetes 46:653-658

26. Torres SH, De Sanctis JB, de LBM, Hernandez N, Finol HJ (2004) Inflammation and nitric oxide production in skeletal muscle of type 2 diabetic patients. J Endocrinol 181:419-427

27. Tang J, Kern TS (2011) Inflammation in diabetic retinopathy. Prog Retin Eye Res 30:343-358

28. Natali A, Taddei S, Quinones Galvan A et al (1997) Insulin sensitivity, vascular reactivity, and clamp-induced vasodilatation in essential hypertension. Circulation 96:849-855

29. Schmetterer L, Muller M, Fasching P et al (1997) Renal and ocular hemodynamic effects of insulin. Diabetes 46:1868-1874

30. Manrique C, Lastra G, Sowers JR (2014) New insights into insulin action and resistance in the vasculature. Ann N Y Acad Sci 1311:138-150

31. Zeng G, Quon MJ (1996) Insulin-stimulated production of nitric oxide is inhibited by wortmannin. Direct measurement in vascular endothelial cells. J Clin Invest 98:894-898

32. Muniyappa R, Sowers JR (2013) Role of insulin resistance in endothelial dysfunction. Rev Endocr Metab Disord 14:5-12

33. Eghbalzadeh K, Brixius K, Bloch W, Brinkmann C (2014) Skeletal muscle nitric oxide (NO) synthases and NO-signaling in "diabesity" - what about the relevance of exercise training interventions? Nitric Oxide 37:28-40

34. Liew G, Sharrett AR, Wang JJ et al (2008) Relative importance of systemic determinants of retinal arteriolar and venular caliber: the atherosclerosis risk in communities study. Arch Ophthalmol 126:1404-1410

35. Klein R, Klein BE, Moss SE et al (2004) The relation of retinal vessel caliber to the incidence and progression of diabetic retinopathy: XIX: the Wisconsin Epidemiologic Study of Diabetic Retinopathy. Arch Ophthalmol 122:76-83

36. Mandecka A, Dawczynski J, Vilser W et al (2009) Abnormal retinal autoregulation is detected by provoked stimulation with flicker light in well-controlled patients with type 1 diabetes without retinopathy. Diabetes Res Clin Pract 86:51-55

37. Nguyen TT, Wang JJ, Wong TY (2007) Retinal vascular changes in pre-diabetes and prehypertension: new findings and their research and clinical implications. Diabetes Care 30:2708-2715

38. Stern SE, Williams K, Ferrannini E, DeFronzo RA, Bogardus C, Stern MP (2005) Identification of individuals with insulin resistance using routine clinical measurements. Diabetes 54:333-339 